\title{
Three-dimensional simulated microgravity culture improves the proliferation and odontogenic differentiation of dental pulp stem cell in PLGA scaffolds implanted in mice
}

\author{
YANPING LI*, LINA HE* , SHUANG PAN, LIN ZHANG, WEIWEI ZHANG, HONG YI and YUMEI NIU \\ Department of Endodontics, The First Affiliated Hospital of Harbin Medical University, \\ Harbin, Heilongjiang 150001, P.R. China
}

Received November 24, 2015; Accepted November 17, 2016

DOI: $10.3892 / \mathrm{mmr} .2016 .6042$

\begin{abstract}
Tooth regeneration through stem cell-based therapy is a promising treatment for tooth decay and loss. Human dental pulp stem cells (hDPSCs) have been widely identified as the stem cells with the most potential for tooth tissue regeneration. However, the culture of hDPSCs in vitro for tissue engineering is challenging, as cells may proliferate slowly or/and differentiate poorly in vivo. Dynamic three-dimensional (3D) simulated microgravity (SMG) created using the rotary cell culture system is considered to an effective tool, which contributes to several cell functions. Thus, the present study aimed to investigate the effect of dynamic 3D SMG culture on the proliferation and odontogenic differentiation abilities of hDPSCs in poly (lactic-co-glycolic acid) (PLGA) scaffolds in nude mice. The hDPSCs on PLGA scaffolds were maintained separately in the 3D SMG culture system and static 3D cultures with osteogenic medium for 7 days in vitro. Subsequently, the cell-PLGA complexes were implanted subcutaneously on the backs of nude mice for 4 weeks. The results of histological and immunohistochemical examinations of Ki-67, type I collagen, dentin sialoprotein and DMP-1 indicated that the proliferation and odontogenic differentiation abilities of the hDPSCs prepared in the 3D SMG culture system were higher, compared with those prepared in the static culture system. These findings suggested that dynamic 3D SMG culture likely contributes to tissue engineering by improving the proliferation and odontogenic differentiation abilities of hDPSCs in vivo.
\end{abstract}

Correspondence to: Professor Yumei Niu, Department of Endodontics, The First Affiliated Hospital of Harbin Medical University, 143 Yiman Street, Harbin, Heilongjiang 150001, P.R. China

E-mail: yumeiniu@163.com

${ }^{*}$ Contributed equally

Key words: simulated microgravity, dental pulp stem cell, poly (lactic-co-glycolic acid) scaffolds, proliferation, odontogenic differentiation

\section{Introduction}

Tooth loss due to periodontal disease, dental caries or trauma affects the quality of life of an individual. Attempts to successfully regenerate lost teeth or their components have long been an ambition of dentists. Based on stem cells, scaffolds and growth factors for regenerating missing or damaged tissues, tissue engineering is one of the latest emerging innovations, aimed at providing solutions for tissue creation and repair (1). Dental pulp stem cells (DPSCs) are characterized by their multipotent differentiation, self-renewal ability, clonogenic capacity and their odontogenic differentiation potential in particular. Previous studies have shown that DPSCs are capable of differentiating into odontoblast-like cells in vitro, and to form the dentin-pulp-like complex when transplanted into immunocompromised mice in vivo (2). However, despite the promising characteristics of hDPSCs, there are certain challenges, which require addressing prior to the routine use of regenerative techniques involving these cells in clinical applications, including improving the proliferation capacity and committed differentiation efficiency of the cells in biomaterials.

Scaffolds are indispensable in tissue engineering, as they serve as carriers to facilitate the delivery of stem cells and/or growth factors at a three-dimensional (3D) site to guide tissue formation by mediating cell survival and cell-scaffold interactions. Owing to their biodegradability, biocompatibility, and their nontoxic and nonimmunogenic properties (3-5), polymers are widely used in medical applications. Poly (lactic-co-glycolic acid) (PLGA) is a copolymer with desirable physical and mechanical properties. PLGA is a commonly used biomaterial for tissue engineering and is approved for clinical use (6). However, the diffusion of air and nutrient components in conventional $2 \mathrm{D}$ and $3 \mathrm{D}$ static culture is uneven, resulting in reduced cell growth, particularly within the $3 \mathrm{D}$ constructs $(7,8)$.

In previous years, with its low hydrodynamic shear stress and low turbulence, the rotary cell culture system (RCCS) has been shown to allow the exchange of nutrients and transport of cellular secretions (9), contributing to the regulation of the differentiation and proliferation of stem cells. It has been suggested that RCCS provides a more controlled dynamic 3D 
stimulated microgravity (SMG) environment, which qualifies for improved cell-cell interactions, and communication associated with proliferation and differentiation (10-12). Of note, several cell types and tissues have been successfully cultured under 3D SMG conditions, including the formation of living organoid-like tissue architecture, for example, cartilage and bone, in vitro (13-16). However, there have been few repots on the proliferation and differentiation of undifferentiated cells in vivo following culture in 3D SMG.

The present study investigated the proliferation and odontogenic differentiation of hDPSCs in vivo following the use of a 3D SMG culture system compared with static 3D culture. The isolated and identified hDPSCs seeded in PLGA scaffolds were maintained separately in the 3D SMG culture system and the static 3D culture system with osteogenic medium for 7 days in vitro. Subsequently, the differentiating cells with scaffolds were implanted subcutaneously on the backs of nude mice for 6 weeks. Histological and immunohistochemical analyses indicated that the proliferation and odontogenic differentiation abilities of the hDPSCs prepared in the 3D SMG culture system were higher, compared with those prepared in static culture. These results demonstrated the advantages of the 3D SMG culture system for improving the proliferation and odontogenic differentiation abilities of hDPSCs in vivo.

\section{Materials and methods}

Isolation and identification of hDPSCs. All experiments performed in the present study were approved by the Ethics Committee of the First Affiliated Hospital of Harbin Medical University (Harbin, China). The isolation and culturing of the cells were performed as described previously (2). In brief, fresh dental pulp tissues were isolated from healthy impacted third molars of donors (age range, 18-29 years) from the department of the Oral and Maxillofacial Surgery of the First Affiliated Hospital of Harbin Medical University (Harbin, China) in July 2014, following the provision of written informed consent. The dental pulp tissues were digested with $3 \mathrm{mg} / \mathrm{ml}$ collagenase type I (Sigma-Aldrich; Merck Millipore, Darmstadt,) and $4 \mathrm{mg} / \mathrm{ml}$ dispase (BD Biosciences, San Jose, CA, USA) for $1 \mathrm{~h}$ at $37^{\circ} \mathrm{C}$, following which the solution was passed through a $70-\mu \mathrm{m}$ strainer. The characterizations of the hDPSCs were based on a previous report (17). The cells between passages two and five were used in the following experiments.

Cell-PLGA complex culture. The PLGA scaffolds (Synthecon, Inc., Houston, TX, USA) were pretreated, as previously described (17). The hDPSCs $\left(2 \times 10^{6}\right)$ were seeded into each scaffold and cultured in Dulbecco's modified Eagle's medium (DMEM; Hyclone; GE Healthcare Life Sciences, Chalfont, UK) supplemented with $10 \%$ fetal bovine serum (FBS; Hyclone; GE Healthcare Life Sciences) for $72 \mathrm{~h}$ at $37^{\circ} \mathrm{C}$. Subsequently, the cell-scaffold composites were randomly divided into two groups: Static 3D culture and 3D SMG culture. The 3D SMG group was transferred into a 55-ml high-aspect-ratio vessel (Synthecon, Inc.) filled with osteogenic medium (DMEM supplemented with 10\% FBS, $10 \mathrm{nM}$ dexamethasone, $10 \mathrm{mM}$ $\beta$-glycerophosphate and $50 \mu \mathrm{g} / \mathrm{ml}$ ascorbic acid) in the RCCS (Synthecon, Inc.). The rotation speed of the vessel was adjusted throughout the period of cultivation to maintain the complexes at a relatively steady position within the vessel. In parallel, cells cultured in static culture with osteogenic medium were used as controls.

Scanning electron microscopy (SEM) observation. The cell-scaffold complexes of the static 3D culture and 3D SMG culture systems were gently rinsed three times with PBS. The samples were then fixed with $2 \%$ glutaraldehyde and dehydrated using a graded ethanol series of 30, 50, 70, 90 and $100 \%$. Following being dipped into isoamyl acetate and dried in a critical-point dryer, the samples were observed under an SEM.

In vivo transplantation. A total of 20 female nude mice (6-8 weeks old) (Weitonglihua Experimental Animal Technology Co., Ltd., Beijing, China) were randomly assigned into the two groups. All the animals were housed under standard conditions of $12 \mathrm{~h}$ light/dark cycles and fed an autoclaved laboratory rodent diet. Following culture in the 3D static or 3D SMG rotating culture systems with osteogenic medium for 7 days in vitro, the cell-PLGA complexes were implanted subcutaneously onto the backs of the nude mice for 4 weeks. In each mouse, one control scaffold and one SMG scaffold was present on either side of the spine. At 4 weeks post-transplantation, the mice were sacrificed by cervical dislocation followed by extraction of the implants. The implants were fixed in formalin, embedded in paraffin and cut into sections measuring $5 \mu \mathrm{m}$ in thickness for histological and immunohistochemical examinations.

Histology. The sample sections were deparaffinized in xylene, rehydrated through a gradient of ethanol solutions, stained with hematoxylin and eosin (H\&E), Masson's trichrome staining and von Kossa staining, and viewed using a light microscope (Olympus Corporation, Tokyo, Japan).

Immunohistochemistry. Immunohistochemical analyses of the retrieved implants were performed using the streptavidin-biotin complex method, according to the manufacturer's recommended protocol. The deparaffinized sections were treated with $100 \mu \mathrm{l} 3 \% \mathrm{H}_{2} \mathrm{O}_{2}$ for $10 \mathrm{~min}$ at room temperature to suppress endogenous peroxidase activity. The sections were then blocked in 5\% normal goat serum (Beijing Zhongshan Golden Bridge Biotechnology, Co.; OriGene Technologies, Inc., Rockville, MD, USA) for $1 \mathrm{~h}$ at room temperature and incubated with primary antibodies (1:100-1:500 dilutions) overnight at $4{ }^{\circ} \mathrm{C}$. The following primary monoclonal antibodies were used: Ki-67 and type I collagen (rabbit anti-mouse, cat. no. ab16667, diluted 1:500 and goat anti-mouse, cat. no. ab34710, diluted 1:100; Abcam, Cambridge, MA USA), and dentin sialoprotein (DSP) and DMP-1 (goat anti-mouse, cat. nos. sc-18328 and sc-54181, diluted 1:100 and 1:200; Santa Cruz Biotechnology, Inc. Dallas, TX, USA). Incubation in PBS alone instead of primary antibodies served as negative controls. The sections were rinsed in PBST and incubated in biotinylated secondary antibodies (anti-goat $\mathrm{IgG}$, cat. no. sc-2042 and anti-rabbit IgG, cat. no. sc-2040, all purchased from Santa Cruz Biotechnology, Inc.; diluted 1:400) for $45 \mathrm{~min}$ at room temperature. The sections were then washed three times in PBST, incubated in streptavidin-biotin complex 
A

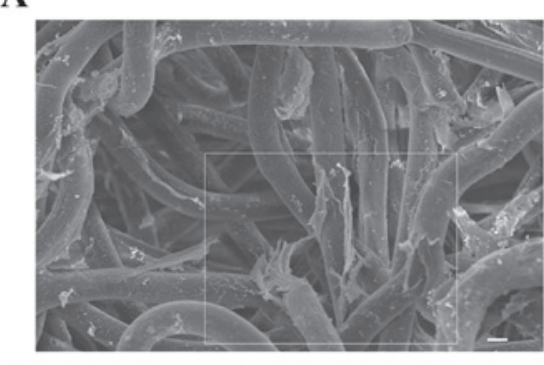

C

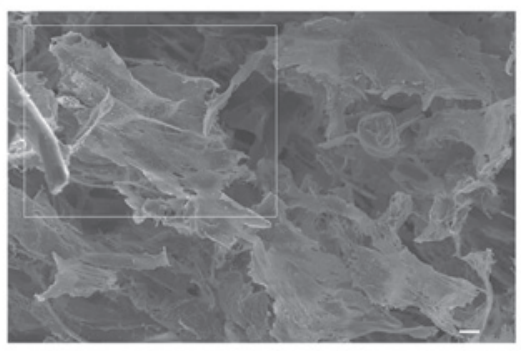

B

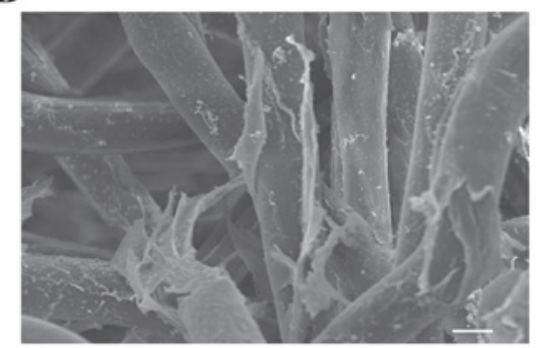

D

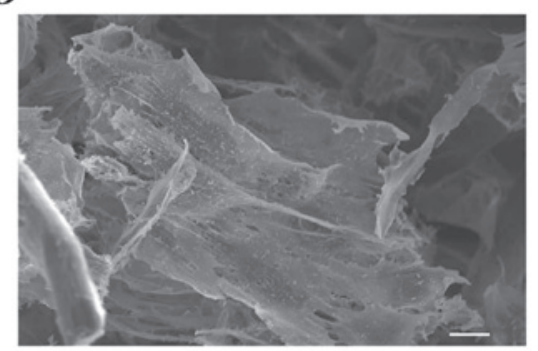

Figure 1. Scanning electron microscopic evaluation of hDPSCs in the poly (lactic-co-glycolic acid) scaffold. (A and B) At 7 days post-seeding of hDPSCs onto the scaffold under static 3D cultures, the cells were attached to the inner surface of the scaffold. (C and D) At 7 days post-seeding, the cells in the 3D SMG culture system grew tightly to each other with abundant extracellular matrix deposited on the scaffolds. Scale bar=100 $\mu \mathrm{m}$. hDPSCs, human dental pulp stem cells.

for $30 \mathrm{~min}$ at room temperature and stained with $100 \mu \mathrm{l} \mathrm{DAB}$ solution. When brown coloration was detected, the slides were rinsed and then counterstained with hematoxylin for $1 \mathrm{~min}$ and observed under a light microscope.

Statistical analysis. The numbers of hDPSCs were counted three times $(n=3)$ in each field of view and section, with three samples for each group. Immunohistochemical analyses were performed using three samples for each group, and calculated three times with ImageJ software (National Institutes of Health, Bethesda, MD, USA). Values are presented as the means \pm standard deviation. Statistical analyses were performed using Student's t-test with SPSS version 16.0 software. (SPSS, Inc., Chicago, IL, USA) $\mathrm{P}<0.05$ was considered to indicate a statistically significant difference.

\section{Results}

Cell morphology of hDPSCs in PLGA under static $3 D$ culture and $3 D$ SMG culture in vitro. To investigate the cellular interaction of hDPSC within PLGA scaffolds under static 3D culture and 3D SMG culture, cell growth and morphology were observed using an electron microscope. As shown in Fig. 1A and B, the cells under static 3D culture were attached to the inner surface of the scaffold in vitro. The cells in the 3D SMG culture system grew tightly to each other with abundant extracellular matrix deposited on the scaffolds (Fig. 1C and D). These results indicated that the scaffolds were suitable for the following in vivo experiments.

$3 D$ SMG culture promotes the growth of hDPSCs in vivo. Following 3D static or 3D SMG culture for 7 days with osteogenic medium in vitro, the differentiating cells within the scaffolds were implanted subcutaneously on the backs of nude mice. Subsequent H\&E staining showed that the number of cells cultured in the SMG system was higher, compared with that in the static culture system (Fig. 2A and B). Immunohistochemical analysis of the endogenous proliferation marker, Ki-67, showed an increase in cell proliferation in the SMG group (Fig. 2C and D).

3D SMG culture induces increased collagen fibrils, calcium phosphate formation and the expression of DMP-1 and dentin sialoprotein (DSP) in vivo. The tissue sections were stained with Masson's trichrome and von Kossa to identify evidence of collagen fiber formation and mineralization, respectively. The Masson's trichrome staining showed a higher number of collagen fibers stained blue in the SMG culture, compared with the static culture (Fig. 3A). An increase of von Kossa staining was observed in the SMG culture, suggesting that the 3D SMG culture enhanced matrix mineralization. The positive staining of type I collagen observed in the static culture was also reduced, compared with the SMG culture, consistent with the results of the Masson's trichrome staining (Fig. 3A). The immunohistochemical data showed a marginal increase in the expression of DMP-1 (Fig. 3B). However, a significant increase in the expression of DSP was observed in the 3D SMG culture, compared with the static culture (Fig. 3C).

\section{Discussion}

In the present study, a 3D dynamic system consisting of an SMG rotary bioreactor, biodegradable polymer scaffolds and osteogenic medium, was successfully established. Following the culture of DPSCs in this system for 7 days in vitro, post-transplantation analysis indicated that the proliferation and odontogenic differentiation abilities of the hDPSCs were increased compared with those of cells cultured in the static culture system. These findings indicated that the 3D SMG 
A

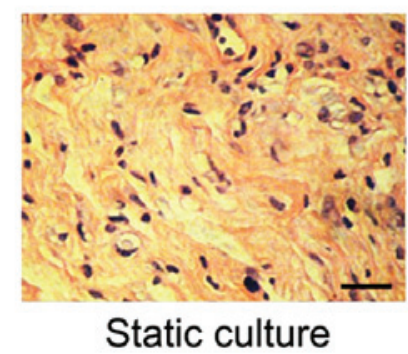

C

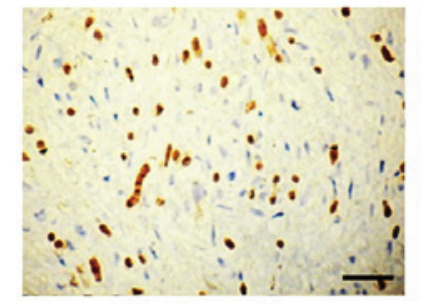

Static culture

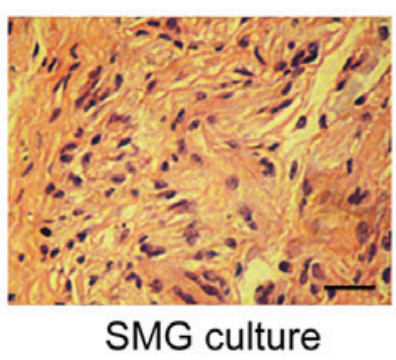

SMG culture

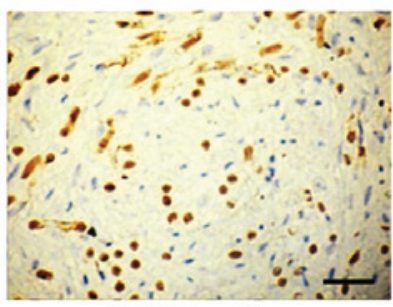

SMG culture
B

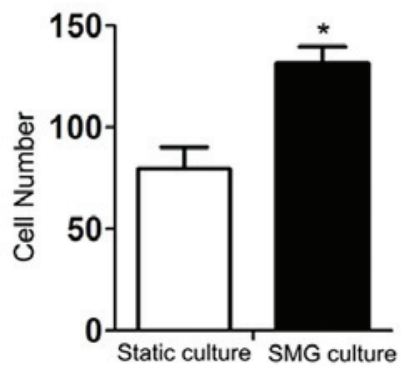

D

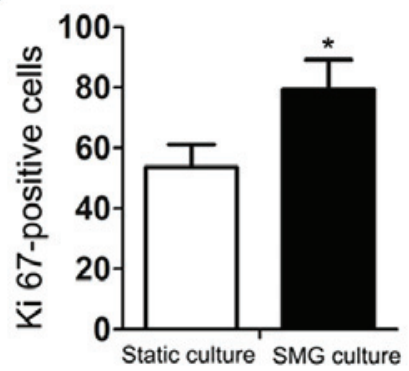

Figure 2. Effects of 3D SMG culture on the growth of hDPSCs in vivo. (A) Hematoxylin and eosin staining of cells grown on scaffolds transplanted on the back of nude mice for $72 \mathrm{~h}$. Following 3D static or 3D SMG culture for 7 days with osteogenic medium, the (B) number of cells in the SMG culture was higher, compared with that in the static culture. (C) Images and (D) quantification of the immunohistochemical analysis of the Ki-67 showed increased positive staining in the SMG cultures, compared with the static cultures ( $\mathrm{P}<0.05)$. Scale bar $=10 \mu \mathrm{m}$. SMG, simulated microgravity; hDPSCs, human dental pulp stem cells.

A
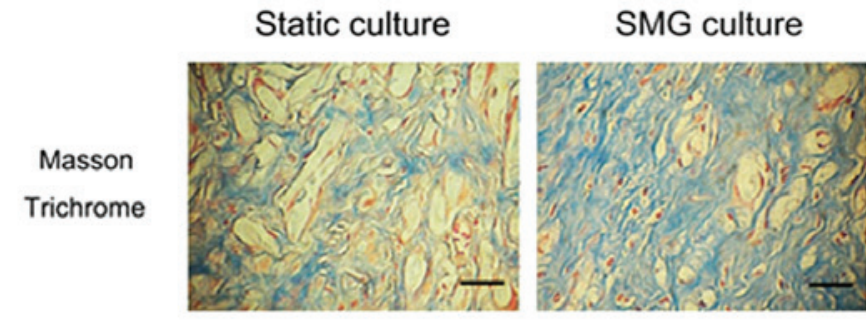

von Kossa
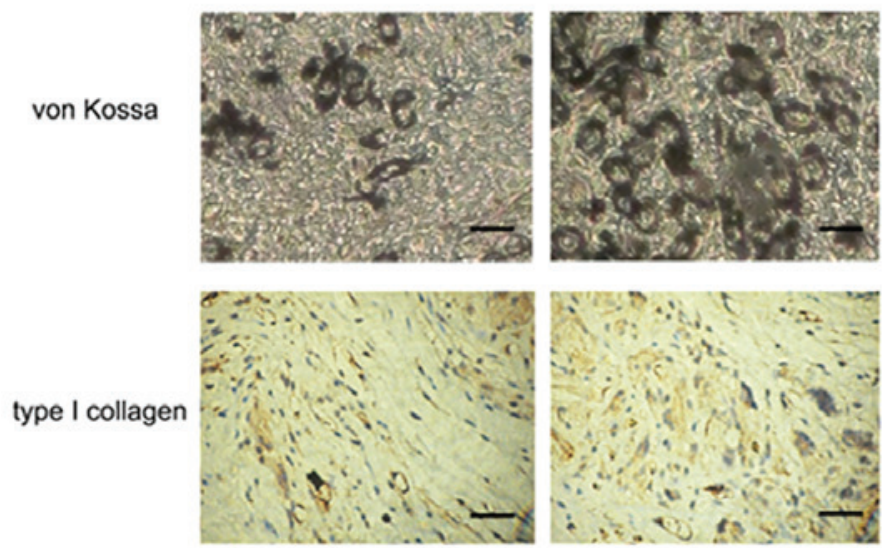

B
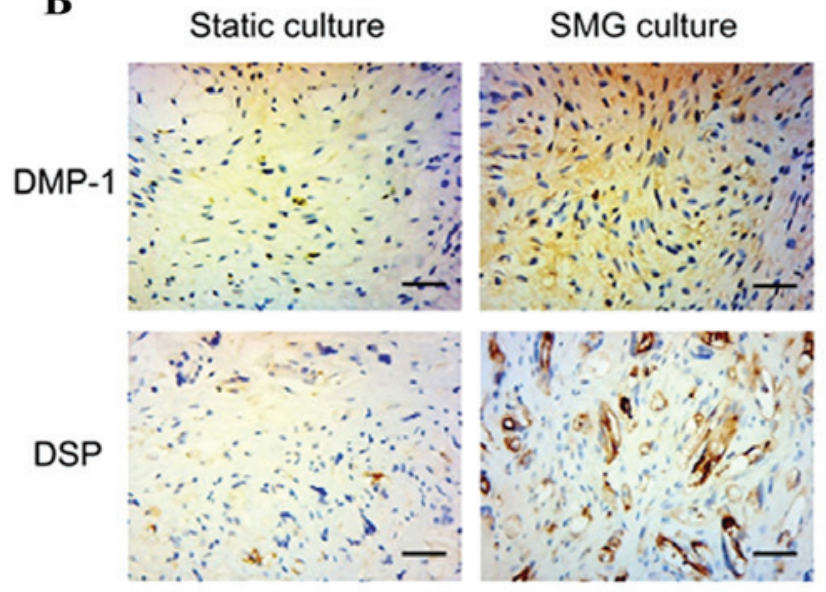

C

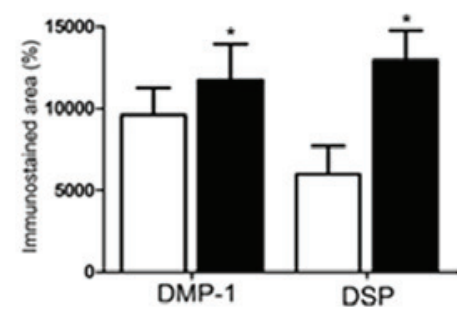

$\square$ Static culture

Figure 3. Effects of 3D SMG culture on the odontogenic differentiation of hDPSCs in vivo. (A) Masson's trichrome staining revealed increased collagen fibers stained blue in the SMG culture, compared with the static culture. Increased von Kossa staining was observed in the SMG culture. Positive staining of type I collagen in the static cultures was lower, compared with than that in the SMG cultures. (B) Immunohistochemical data showed a marginal increase in the expression of DMP-1. The expression of DSP was significantly higher in the 3D SMG culture, compared to static cultures. (C) Areas of immunostaining of each protein were detected ( $\mathrm{P}<0.05)$. Scale bar $=10 \mu \mathrm{m}$. SMG, simulated microgravity; hDPSCs, human dental pulp stem cells; DMP-1, dentin matrix acidic phosphoprotein 1; DSP, dentin sialoprotein.

dynamic system offers potential for use as a potent method for tooth tissue regeneration.
Due to their odontogenic differentiation potential, DPSCs been used as effective seed cells for dental tissue engineering 
and regeneration. The application of DPSCs in dental tissue engineering provides a significant enhancement to dental regeneration; however, sufficient cell numbers are required, leading to the formation of 3D mineralized, dentin tissue-like constructs. However, limitations in the quantity and committed differentiation efficiency of DPSCs inevitably introduce challenges to dental regeneration. Microgravity and polymer scaffolds have been confirmed to offer significant advantages in cell culture by providing a dynamic 3D microenvironment with low-shear forces and high-mass transfer (18-23).

Previous studies have indicated that a variety of 3D biomaterials are suitable for the proliferation and differentiation of DPSCs (24-26). However, due to the effect of gravity, cells seeded in $3 \mathrm{D}$ static culture systems preferentially fall down to the base of the scaffolds, rather than scattering evenly (27). In addition, air, nutrient components and metabolic wastes are also distributed unevenly. Cellular metabolic waste is difficult to transport out of the scaffolds, and the concentration of growth/differentiation factors is usually confined to the surface of the scaffold, resulting in decreased cell proliferation and lineage-specific differentiation $(28,29)$. Fortunately, these problems can be overcome by the dynamic system of the SMG rotary bioreactor, which creates a suspension culture environment contributing to supply of oxygen and nutrients, and the transport of metabolic waste from the cells. In the present study, a 3D dynamic system of SMG was prepared for 7 days in vitro, in which the DPSCs grew tightly to each other with abundant extracellular matrix, and a higher number of cells were observed in vivo (Fig. 2). As previous experiments have reported, undergoing 3D dynamic SMG culture leads to the promotion of cell proliferation (18,30-32). Ki-67, used as a biomarker for the proliferation of cells, showed an increase in cell proliferation in the dynamic system (Fig. 2) as a result of DPSCs obtaining sufficient nutrition and the prompt delivery of metabolic waste in the suspension culture environment. Following transplantation in vivo, the optimal viability and state of the DPSCs were observed. The increased proliferation of the DPSCs in vivo is important for dental tissue engineering and regeneration, as this is limited in autologous or allogenic seeding of cells.

The most notable feature of hDPSCs is their odontogenic differentiation potential for dental tissue engineering (33). In the present study, Masson's trichrome staining and the immunohistochemical analysis of type I collagen were applied to determine the collagen fibers in the tissue sections. Type I collagen is the most important constituent of the extracellular matrix of dental pulp connective tissue (34). It has been suggested that the synthesis of type I collagen is an important step in the odontoblast differentiation process (35). Previous studies have shown that type I collagen may be a component of the predentin secreted by polarized odontoblasts (36), and it has been found to be associated with the production and mineralization of dentine (37). In the present study, increased collagen was produced in the dynamic system group, which indicated that dynamic culture triggered the deposition of oriented collagen fibers, which in turn suggested the possibility of the formation of dentin. Von Kossa staining is usually used to identify the existence and formation of calcium phosphate (38). As the primary component of teeth is calcium phosphate, the results indicating a higher level of calcium phosphate formation in the implanted cells from the dynamic SMG system, compared with that in static culture 4 weeks post-transplantation ex vivo suggested that the dynamic SMG system promoted the mineralization of DPSCs. DSP and DMP-1, the major noncollagenous proteins synthesized by odontoblasts, are well-known markers of odontogenic differentiation. DSP, which is expressed at high levels in odontoblasts, is essential to the formation and calcification of dentin $(39,40)$. Expressed prior to DSP, DMP-1 regulates the mineralization of dentin (41) and is involved in the differentiation of odontoblasts (42-44). Thus DSP and DMP-1 are usually selected as specific markers of differentiation to detect the odontogenic potential of DPSCs. The upregulation of DSP and DMP-1 in the DPSCs induced by the dynamic SMG system, indicated the promotion of odontogenesis of the DPSCs. The dynamic system of SMG upregulated the mineralization capacity and expression levels of DSP and DMP-1 in the DPSCs, which supported the idea that the dynamic SMG system was more suitable for odontogenic differentiation of DPSCs. There is substantial evidence, which shows that SMG promotes the differentiation of stem cells in vitro (18,30-32) and, consistent with these reports, the present study found that the dynamic SMG system increased the odontogenic differentiation of DPSCs in vivo. This may also be due, in part, to the prompt delivery of factors in osteogenic medium and interactions with the microenvironment in the nude mice. The DPSCs under the dynamic culture system, which contributed to the sufficient transfer of nutrients and factors in osteogenic medium, were maintained in good condition throughout the entire treatment process prior to in vivo implantation. With DPSCs in a preferable condition, the interaction between cells and the microenvironment in vivo may be improved, which may have a positive effect on the committed differentiation of stem cells.

In conclusion, the present study showed that the dynamic system combining SMG with scaffolds and osteogenic medium significantly improved the proliferation and odontogenic differentiation of DPSCs by improving their metabolism and microenvironment. These results further indicated the potential of the dynamic SMG system in dentin regeneration, and provided novel insight into tooth engineering.

\section{Acknowledgements}

This study was supported by grants from The Nature Science Foundation of China (grant nos. 81271132 and 81570963) and the Nature Science Foundation of Heilongjiang Province (grant no. H201440).

\section{References}

1. Langer R and Vacanti JP: Tissue engineering. Science 260: 920-926, 1993

2. Gronthos S, Mankani M, Brahim J, Robey PG and Shi S: Postnatal human dental pulp stem cells (DPSCs) in vitro and in vivo. Proc Natl Acad Sci USA 97: 13625-13630, 2000.

3. Rutzky LP, Bilinski S, Kloc M, Phan T, Zhang H, Katz SM and Stepkowski SM: Microgravity culture condition reduces immunogenicity and improves function of pancreatic islets1. Transplantatio 74: 13-21, 2002.

4. Vilos C and Velasquez LA: Therapeutic strategies based on polymeric microparticles. J Biomed Biotechnol 2012: 672760, 2012.

5. Lesman A, Koffler J, Atlas R, Blinder YJ, Kam Z and Levenberg S: Engineering vessel-like networks within multicellular fibrin-based constructs. Biomaterials 32: 7856-7869, 2011. 
6. Lü JM, Wang X, Marin-Muller C, Wang H, Lin PH, Yao Q and Chen C: Current advances in research and clinical applications of PLGA-based nanotechnology. Expert Rev Mol Diagn 9: 325-341, 2009

7. Holy CE, Shoichet MS and Davies JE: Engineering three-dimensional bone tissue in vitro using biodegradable scaffolds: Investigating initial cell-seeding density and culture period. J Biomed Mater Res 51: 376-382, 2000.

8. Inanc B, Elcin AE and Elcin YM: Osteogenic induction of human periodontal ligament fibroblasts under two- and three-dimensional culture conditions. Tissue Eng 12: 257-266, 2006.

9. Rutzky LP, Bilinski S, Kloc M, Phan T, Zhang H, Katz SM and Stepkowski SM: Microgravity culture condition reduces immunogenicity and improves function of pancreatic islets1. Transplantation 74: 13-21, 2002.

10. Hammond TG and Hammond JM: Optimized suspension culture: The rotating-wall vessel. Am J Physiol Renal Physiol 281: F12-F25, 2001.

11. Klement BJ, Young QM, George BJ and Nokkaew M: Skeletal tissue growth, differentiation and mineralization in the NASA rotating wall vessel. Bone 34: 487-498, 2004.

12. Goodwin TJ, Prewett TL, Wolf DA and Spaulding GF: Reduced shear stress: A major component in the ability of mammalian tissues to form three-dimensional assemblies in simulated microgravity. J Cell Biochem 51: 301-311, 1993.

13. Ohyabu Y, Kida N, Kojima H, Taguchi T, Tanaka J and Uemura T: Cartilaginous tissue formation from bone marrow cells using rotating wall vessel (RWV) bioreactor. Biotechnol Bioeng 95: 1003-1008, 2006.

14. Qiu QQ, Ducheyne P and Ayyaswamy PS: Fabrication, characterization and evaluation of bioceramic hollow microspheres used as microcarriers for 3-D bone tissue formation in rotating bioreactors. Biomaterials 20: 989-1001, 1999.

15. Khaoustov VI, Darlington GJ, Soriano HE, Krishnan B, Risin D, Pellis NR and Yoffe B: Induction of three-dimensional assembly of human liver cells by simulated microgravity. In Vitro Cell Dev Biol Anim 35: 501-509, 1999

16. Martin A, Zhou A, Gordon RE, Henderson SC, Schwartz AE, Schwartz AE, Friedman EW and Davies TF: Thyroid organoid formation in simulated microgravity: Influence of keratinocyte growth factor. Thyroid 10: 481-487, 2000

17. He L, Pan S, Li Y, Zhang L, Zhang W, Yi H, Song C and Niu Y: Increased proliferation and adhesion properties of human dental pulp stem cells in PLGA scaffolds via simulated microgravity. Int Endod J 49: 161-173, 2016.

18. Lei XH, Ning LN, Cao YJ, Liu S, Zhang SB, Qiu ZF, Hu HM, Zhang HS, Liu S and Duan EK: NASA-approved rotary bioreactor enhances proliferation of human epidermal stem cells and supports formation of 3D epidermis-like structure. PLoS One 6 : e26603, 2011.

19. Wu C, Guo X, Wang F, Li X, Tian XC, Li L, Wu Z and Zhang S: Simulated microgravity compromises mouse oocyte maturation by disrupting meiotic spindle organization and inducing cytoplasmic blebbing. PLoS One 6: e22214, 2011.

20. Freed LE, Hollander AP, Martin I, Barry JR, Langer R and Vunjak-Novakovic G: Chondrogenesis in a cell-polymer-bioreactor system. Exp Cell Res 240: 58-65, 1998.

21. Pan $\mathrm{H}$, Jiang $\mathrm{H}$ and Chen W: Interaction of dermal fibroblasts with electrospun composite polymer scaffolds prepared from dextran and poly lactide-co-glycolide. Biomaterials 27: 3209-3220, 2006

22. McBane JE, Battiston KG, Wadhwani A, Sharifpoor S, Labow RS and Santerre JP: The effect of degradable polymer surfaces on co-cultures of monocytes and smooth muscle cells. Biomaterials 32: 3584-3595, 2011

23. Xue $\mathrm{Y}$, Dånmark $\mathrm{S}$, Xing $\mathrm{Z}$, Arvidson $\mathrm{K}$, Albertsson $\mathrm{AC}$ Hellem S, Finne-Wistrand A and Mustafa K: Growth and differentiation of bone marrow stromal cells on biodegradable polymer scaffolds: An in vitro study. J Biomed Mater Res A 95: $1244-1251,2010$

24. Karadzic I, Vucic V, Jokanovic V, Debeljak-Martacic J, Markovic D, Petrovic S and Glibetic M: Effects of novel hydroxyapatite-based 3D biomaterials on proliferation and osteoblastic differentiation of mesenchymal stem cells. J Biomed Mater Res A 103: $350-357,2015$.
25. Miyashita S, Ahmed NE, Murakami M, Iohara K, Yamamoto T, Horibe H, Kurita K, Takano-Yamamoto T and Nakashima M: Mechanical forces induce odontoblastic differentiation of mesenchymal stem cells on three-dimensional biomimetic scaffolds J Tissue Eng Regen Med: Jun 12, 2014 (Epub ahead of print).

26. Roozafzoon R, Lashay A, Vasei M, Ai J, Khoshzaban A, Keshel SH, Barabadi $\mathrm{Z}$ and Bahrami H: Dental pulp stem cells differentiation into retinal ganglion-like cells in a three dimensional network. Biochem Biophys Res Commun 457: 154-160, 2015.

27. Boukhechba F, Balaguer T, Michiels JF, Ackermann K, Quincey D, Bouler JM, Pyerin W, Carle GF and Rochet N: Human primary osteocyte differentiation in a 3D culture system. J Bone Miner Res 24: 1927-1935, 2009.

28. Hassell T, Gleave S and Butler M: Growth inhibition in animal cell culture. The effect of lactate and ammonia. Appl Biochem Biotechnol 30: 29-41, 1991.

29. Glowacki J, Mizuno S and Greenberger JS: Perfusion enhances functions of bone marrow stromal cells in three-dimensional culture. Cell Transplant 7: 319-326, 1998.

30. Yuge L, Kajiume T, Tahara H, Kawahara Y, Umeda C, Yoshimoto R, Wu SL, Yamaoka K, Asashima M, Kataoka K and Ide T: Microgravity potentiates stem cell proliferation while sustaining the capability of differentiation. Stem Cells Dev 15: 921-929, 2006

31. Li S, Ma Z, Niu Z, Qian H, Xuan D, Hou R and Ni L: NASA-approved rotary bioreactor enhances proliferation and osteogenesis of human periodontal ligament stem cells. Stem Cells Dev 18: 1273-1282, 2009.

32. Qiu Q, Ducheyne P, Gao H and Ayyaswamy P: Formation and differentiation of three-dimensional rat marrow stromal cell culture on microcarriers in a rotating-wall vessel. Tissue Eng 4: 19-34, 1998

33. About I, Bottero MJ, de Denato P, Camps J, Franquin JC and Mitsiadis TA: Human dentin production in vitro. Exp Cell Res 258: 33-41, 2000.

34. Karjalainen S, Söderling E, Pelliniemi L and Foidart JM Immunohistochemical localization of types I and III collagen and fibronectin in the dentine of carious human teeth. Arch Oral Biol 31: 801-806, 1986.

35. Andujar MB, Couble P, Couble ML and Magloire H: Differential expression of type I and type III collagen genes during tooth development. Development 111: 691-698, 1991.

36. Mao YQ, Ohsaki Y and Kurisu K: Immunohistochemical study of the relationship between extracellular matrix and root bifurcation in the mouse molar. Arch Oral Biol 35: 583-591, 1990.

37. Garcia JM, Martins MD, Jaeger RG and Marques MM: Immunolocalization of bone extracellular matrix proteins (type I collagen, osteonectin and bone sialoprotein) in human dental pulp and cultured pulp cells. Int Endod J 36: 404-410, 2003.

38. Hao J, Narayanan K, Ramachandran A, He G, Almushayt A, Evans $\mathrm{C}$ and George A: Odontoblast cells immortalized by telomerase produce mineralized dentin-like tissue both in vitro and in vivo. J Biol Chem 277: 19976-19981, 2002.

39. McKnight DA, Simmer JP, Hart PS, Hart TC and Fisher LW: Overlapping DSPP mutations cause dentin dysplasia and dentinogenesis imperfecta. J Dent Res 87: 1108-1111, 2008

40. Lee SK, Lee KE, Jeon D, Lee G, Lee H, Shin CU, Jung YJ, Lee SH, Hahn SH and Kim JW: A novel mutation in the DSPP gene associated with dentinogenesis imperfecta type II. J Dent Res 88: 51-55, 2009 .

41. He G, Dahl T, Veis A and George A: Dentin matrix protein 1 initiates hydroxyapatite formation in vitro. Connect Tissue Res 44 (Suppl 1): 240-245, 2003.

42. Almushayt A, Narayanan K, Zaki AE and George A: Dentin matrix protein 1 induces cytodifferentiation of dental pulp stem cells into odontoblasts. Gene Ther 13: 611-620, 2006.

43. Narayanan K, Srinivas R, Ramachandran A, Hao J, Quinn B and George A: Differentiation of embryonic mesenchymal cells to odontoblast-like cells by overexpression of dentin matrix protein 1. Proc Natl Acad Sci USA 98: 4516-4521, 2001.

44. Chaussain C, Eapen AS, Huet E, Floris C, Ravindran S, Hao J, Menashi S and George A: MMP2-cleavage of DMP1 generates a bioactive peptide promoting differentiation of dental pulp stem/progenitor cell. Eur Cell Mater 18: 84-95, 2009. 